\title{
Cryptanalyzing the Polynomial-Reconstruction Based Public-Key System Under Optimal Parameter Choice
}

\author{
Aggelos Kiayias $^{1}$ and Moti Yung ${ }^{2}$ \\ 1 Department of Computer Science and Engineering, \\ University of Connecticut, Storrs, CT, USA \\ aggelos@cse.uconn.edu \\ 2 Department of Computer Science, \\ Columbia University, New York, NY, USA \\ moti@cs.columbia.edu
}

\begin{abstract}
Recently, Augot and Finiasz presented a coding theoretic public key cryptosystem that suggests a new approach for designing such systems based on the Polynomial Reconstruction Problem. Their cryptosystem is an instantiation of this approach under a specific choice of parameters which, given the state of the art of coding theory, we show in this work to be sub-optimal. Coron showed how to attack the Augot and Finiasz cryptosystem. A question left open is whether the general approach suggested by the cryptosystem works or not. In this work, we show that the general approach (rather than only the instantiation) is broken as well. Our attack employs the recent powerful list-decoding mechanisms.
\end{abstract}

\section{Introduction}

Recently, in Eurocrypt 2003 AF03, Augot and Finiasz presented a public-key cryptosystem that was based on the Polynomial Reconstruction problem (PR). This scheme suggests a general approach for designing such cryptosystems; their cryptosystem is an instantiation of this approach based on a specific choice of parameters.

Let us first review PR, which is a curve-fitting problem that has been studied extensively especially in the coding theoretic setting, where it corresponds to the Decoding Problem of Reed-Solomon Codes.

Definition 1 (Polynomial Reconstruction (PR)). Given a set of points over a finite field $\left\{\left\langle z_{i}, y_{i}\right\rangle\right\}_{i=1}^{n}$, and parameters $[n, k, w]$, recover all polynomials $p$ of degree less than $k$ such that $p\left(z_{i}\right) \neq y_{i}$ for at most $w$ distinct indexes $i \in\{1, \ldots, n\}$.

Regarding the solvability of PR, we remark that unique solution can only be guaranteed when $w \leq \frac{n-k}{2}$ (the error-correction bound of Reed-Solomon 
Codes). For such parameter choices, the Berlekamp-Welch Algorithm BW86. can be used to recover the solution in polynomial-time. When the number of errors $w$ exceeds this bound, unique solution is not necessarily guaranteed. In this range, a decoding algorithm may output a list of polynomials that satisfy the constraints. This is called list-decoding and recently some breakthrough results have been achieved in this field. The most powerful list-decoding algorithm is the one by Guruswami and Sudan, GS98. The algorithm will work for any number of errors such that $w<n-\sqrt{(k-1) n}$. For choice of parameters beyond the Guruswami-Sudan solvability bound, no known efficient algorithm exists that solves PR (and GS98] gives some indication why such an algorithm is not likely to be found).
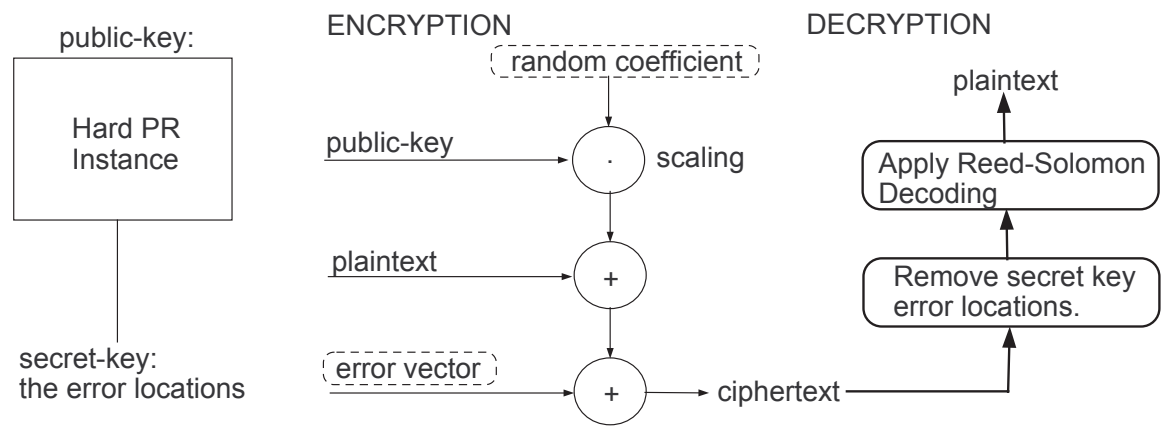

Fig. 1. The Augot and Finiasz general approach for designing a pk-cryptosystem using the hardness of RS decoding

Augot and Finiasz's general approach (see figure 1) is to use a PR instance which is hard to solve (i.e., a highly noisy instance) as a public key, and to encrypt a message by scaling the given public key (i.e., multiplying the polynomial values by a scalar) and adding to the scaled instance the message which is represented as a slightly lower degree second PR instance which is solvable, yielding a PR instance representing the ciphertext. The receiver who knows the noise locations in the public key can recover the message. The approach allows key sizes that are much smaller than the traditional coding theoretic based publickey systems (i.e., the McEliece cryptosystem [McE78]). Further, direct use of the above mentioned decoding and list-decoding methods do not apply to breaking the cryptosystem (directly). To implement the approach of figure 1 one needs to specify: (i) the structure of the public-key, (ii) the structure of the error-vector, and in accordance (iii) the decoding method employed in decryption.

What we noticed is that while the public-key structure was chosen to be an unsolvable PR instance, the choice of the error-vector and the associated decoding method was sub-optimal considering the state-of-the-art of Coding Theory. The scheme was in fact, based on unique decoding (and not list decoding 
techniques) and did not consider probabilistic analysis to maximize the allowed entropy of the error-vector.

The scheme of AF03 was recently broken by Coron Cor03a, Cor03c (without affecting the solvability of PR). The elegant attack presented in Cor03c is in fact a ciphertext-only attack that is built on the Berlekamp-Welch method and recovers the message, given knowledge only of the public-key and a ciphertext. A further modification of the scheme, using extension fields but essentially the same system, was suggested recently AFL03 and was shown by Coron Cor03b to be vulnerable to essentially the same attack.

Coding Theoretic Motivation. The Augot-Finiasz cryptosystem employed unique decoding techniques rather than list-decoding techniques (assuming that unique decoding is what is needed for a correct cryptosystem - an assumption we refute herein). Moreover, they consider only worst-case analysis in the selection of the code parameters. Thus, their cryptosystem is sub-optimal in the above respects given the general approach outlined above.

This leaves open the question of whether this general approach works in principle, i.e., when one uses the optimal coding theoretic techniques and probabilistic analysis for the parameter selection.

Our Results: In this work we investigate the above question. In particular, we maximize the rate of the error vector used during encryption and choose state-of-the-art list-decoding techniques to implement the Reed-Solomon decoding step for decryption. Regarding the optimization (maximization) of the error-rate we make two key observations (1) the system of AF03 employs a worst-case approach in selecting this parameter; a probabilistic approach (that we perform in this work) allows higher values. (2) the system of AF03 employs Berlekamp-Welch RS-decoding for the decryption operation. We emphasize that more powerful decoding techniques can be employed that allow larger values for the error-rate parameter. Our methodology is to use an extended set of tools both for design and analysis in order to get the best possible instantiations of the general approach. The tools include "list decoding" rather than unique decoding techniques (which we show to be still good for decryption, since decoding to a unique value is assured with extremely high probability over a large enough field, even when ambiguous decoding is allowed, cf. Lemma 10.

We develop our presentation as a ping-pong game between a cryptosystems designer and a cryptanalyst. To avoid any misunderstanding our goal is not to design a new cryptosystem, but rather using the design and cryptanalysis steps as a methodology for exploring the general approach.

First Step. Regarding our key-observation (1) we employ the tails of the hypergeometric distribution to show that the original scheme allowed too few errors in the error-vector to be used by the message encryption process. Thus the errorrate can be increased high enough to aid the designer to achieve instances of the cryptosystem where Coron's analysis does not work. But, nevertheless we provide an alternative probabilistic analysis showing that the original attack of 
Coron would work almost always even in this modified (more noisy) version, thus aiding the cryptanalyst.

Second Step. Combining our key-observations (1) and (2) above, we discover the optimal setting for the sender error-parameter ("optimal" under the assumption that the Guruswami-Sudan list-decoding algorithm GS98 represents the best possible decoding algorithm against Polynomial Reconstruction). We show that the optimal parameter setting, helps the designer and in this case Coron's attack fails. To answer our question about the limit of the approach, we then present a new attack that is based on the Sudan and Guruswami-Sudan algorithms Sud97, GS98. Our attack, with overwhelming probability, breaks even the optimal parameter setting. This means that the general approach, outlined in figure 1, taken by Augot and Finiasz (rather than merely their non-optimal instantiation) breaks.

We believe that our results demonstrate how design and analysis of Coding theory based cryptography, must employ probabilistic methods and state of the art decoding techniques. Furthermore, our results and the attack of Coron demonstrate that PR-based cryptosystems that lack formal proofs of security by concrete reduction arguments, even when they seem to be related to PR, are potentially susceptible to coding theoretic attacks that do not imply any weakness in the PR problem itself. Note that, the private-key cryptosystem based on PR suggested by the authors in KY02 was shown to be semantically secure under an intractability decisional assumption that bears upon the average-case PR (for choices of the parameters beyond the Guruswami-Sudan solvability bound). This cryptosystem (as well as the other cryptographic primitives in [KY02] ) are not affected by the techniques of the present paper and of Coron's [Cor03a, Cor03b, Cor03c and breaking these designs seems to require significant advances in RS decodability.

Due to space constraints proofs are omitted from the present abstract; the full-version with all details is available in [KY04.

\section{Background: The Recent Polynomial-Based Public-Key Cryptosystem}

We review the recent developments, while setting up the necessary notations and interesting points regarding our investigation.

\subsection{The Cryptosystem of [AF03]}

The cryptosystem of AF03] can be described in high level as follows:

1. The public-key is a PR-instance of parameters $[n, k+1, W]$ for which (i) the hidden polynomial $p$ is monic; (ii) solving the instance is considered hard. The public-key is a sequence of values in $(\mathbb{F} \times \mathbb{F})^{n}$ (while the locations of the error points is the secret key). 
2. Encryption operates by first transposing (i.e., scaling the polynomial of) the public-key using a random value $\alpha \in \mathbb{F}$ (the encryption coefficient), and then adding to the transposed public-key the message (evaluated as a second polynomial represented as pairs of points using the same first coordinates as the points of the public key PR instance, with no errors), and finally adding some additional $w$ errors. (In other words, the message is embedded in a second PR instance with $w$ errors and added to the transposed public key). It follows that a ciphertext is a sequence of values in $(\mathbb{F} \times \mathbb{F})^{n}$.

3. Decryption removes the points that correspond to public-key errors, i.e., $W$ points of the ciphertext. Decryption relies on the following two facts: (i) the remaining $n-W$ points can be decoded into a polynomial $p^{*}$; (ii) due to the fact that the message polynomial is selected to be of degree less than the degree of the monic polynomial $p$ hidden in the public-key, it follows that the recovery of $p^{*}$ implies the recovery of the encryption coefficient $\alpha$. The message polynomial can be recovered as $p_{m s g}(x)=p^{*}(x)-\alpha p(x)$.

We note that the points over which the polynomials are evaluated in a PR instance can be publicly known (thus the public-key and the ciphertext can be considered to be of size only $\left.|\mathbb{F}|^{n}\right)$.

In more detail, let $z_{1}, \ldots, z_{n} \in \mathbb{F}$ be arbitrary distinct elements of the underlying field, where $n \in \mathbb{N}$ is a security parameter. The public-key of the system is a PR-instance that is generated as follows: first a random tuple $\left\langle E_{1}, \ldots, E_{n}\right\rangle$ is selected that has exactly $W$ non-zero randomly selected elements from $\mathbb{F}$. Second, a random polynomial $p$ of degree less than $k$ is selected. The public-key is set to pk $:=\left\{\left\langle z_{i}, y_{i}\right\rangle\right\}_{i=1}^{n}$ where $y_{i}=p\left(z_{i}\right)+E_{i}+z_{i}^{k}$ for $i=1, \ldots, n$.

Remark. Observe that $\left\{\left\langle z_{i}, y_{i}-z_{i}^{k}\right\rangle\right\}_{i=1}^{n}$ is a random PR-instance with parameters $n, k, W$.

The encryption operation is defined with domain $\mathbb{F}^{k}$ and general range the set $(\mathbb{F} \times \mathbb{F})^{n}$. The message $m s g$ is encoded as a polynomial of degree less than $k$, denoted by $p_{m s g}(x)$; a random tuple $\left\langle e_{1}, \ldots, e_{n}\right\rangle$ is selected so that it has exactly $w$ non-zero randomly selected field elements; a random element $\alpha \in \mathbb{F}$ is selected as well. The ciphertext that corresponds to $m s g$ is the sequence of pairs $\left\{\left\langle z_{i}, y_{i}^{\prime}\right\rangle\right\}_{i=1}^{n}$ defined as follows $y_{i}^{\prime}=\alpha y_{i}+p_{m s g}\left(z_{i}\right)+e_{i}$, for $i=1, \ldots, n$.

So far, the above represents a general approach. The exact choice of parameters (as a function of $n$, say) gives the specific system of [AF03.

The decryption operates as follows: let $I \subseteq\{1, \ldots, n\}$ be such that $|I|=$ $n-W$ and for all $i \in I$ it holds that $E_{i}=0$ (from the selection of the publickey). Observe now that the sequence of pairs $C=\left\{\left\langle z_{i}, y_{i}^{\prime}\right\rangle\right\}_{i \in I}$ can be seen as a PR-instance with parameters $[n-W, k+1, w]$. Now suppose that,

$$
\text { Condition \#1: } \quad w \leq \frac{n-W-k-1}{2} \Rightarrow n \geq 2 w+W+k+1
$$

This condition implies that the PR-instance has a unique solution that can be recovered by the unique decoding technique of Berlekamp-Welch algorithm. 
Given such solution $p^{*}(x)$ it follows that the leading coefficient of $p^{*}$ will be equal to $\alpha$ (by construction, we have that the polynomial hidden into the public-key is monic and of degree $k$ while the degree of the message polynomial is at most $k-1)$. Then, the transmitted message can be recovered as follows $p_{m s g}(x)=$ $p^{*}(x)-\alpha\left(x^{k}+p(x)\right)$.

A second condition is that $W$ should be large, beyond the known bounds of list-decoding, to assure that a third party cannot simply get the error locations of the public key (and thus decrypt all ciphertexts). This condition is the base of the presumed security of the scheme.

\subsection{A Cryptanalytic Framework}

The Cryptanalytic problem that is the basic building block for mounting a ciphertext-only attack on the Public-Key Cryptosystem of AF03. as described above is defined as follows:

Definition 2 Ciphertext-Only Attack Problem (CAP). Given two sequences of tuples $X_{1}:=\left\{\left\langle z_{i}, y_{i}\right\rangle\right\}_{i=1}^{n}$ and $X_{2}:=\left\{\left\langle z_{i}, y_{i}^{\prime}\right\rangle\right\}_{i=1}^{n}$ and parameters $n, k, w, W$ that satisfy the following conditions

i. $w \leq \frac{n-W-k-1}{2}$ and $W \geq n-\sqrt{n(k-1)}$.

ii. $\left\{\left\langle z_{i}, y_{i}-z_{i}^{k}\right\rangle\right\}_{i=1}^{n}$ is a random $P R$-instance with parameters $[n, k, W]$.

iii. $\exists \alpha \in \mathbb{F}$ such that $\left\{\left\langle z_{i}, y_{i}^{\prime}-\alpha y_{i}\right\rangle\right\}_{i=1}^{n}$ is a random PR-instance with parameters $[n, k, w]$.

Goal. Find a list of values of polynomial-length that contains the value $\alpha$.

Any algorithm that solves CAP in polynomial-time can be turned into a ciphertext-only attack against the cryptosystem of AF03, as the following proposition reveals.

Proposition 1. Let $\mathcal{A}$ be an algorithm that solves CAP in polynomial-time. Then any message encrypted in the cryptosystem of [AF03 can be decrypted without knowledge of the secret-key in polynomial-time in the security parameter.

\subsection{Coron's Attack}

In Cor03c, Coron presented an elegant ciphertext-only attack against the cryptosystem of AF03. We explain the attack briefly below and we show that in fact it can be seen as an algorithm to solve CAP (in fact our formulation of CAP above is motivated by the original attack and by further extensions of this idea in the sequel).

Let $X_{1}, X_{2}$ be an instance of CAP, with $X_{1}=\left\{\left\langle z_{i}, y_{i}\right\rangle\right\}_{i=1}^{n} X_{2}=\left\{\left\langle z_{i}, y_{i}^{\prime}\right\rangle\right\}_{i=1}^{n}$ and parameters $k, w, W, n$. Due to condition $i i i$ of definition 2 it follows that there exist $p \in \mathbb{F}[x]$ of degree less than $k$ and $\alpha \in \mathbb{F}$, so that $p\left(z_{i}\right) \neq y_{i}^{\prime}-\alpha y_{i}$ for at most $w$ indexes $i$.

The attack modifies the Berlekamp-Welch algorithm: Let $E(x)$ be a monic polynomial of degree $w$ such that $E\left(z_{i}\right)=0$ for exactly those indexes $i$ for which 
$p\left(z_{i}\right) \neq y_{i}^{\prime}-\alpha y_{i}$. The existence of this polynomial is guaranteed due to the condition iii of definition 2, Let $N(x)=p(x) E(x)$ be a polynomial of degree less than $k+w$.

Now consider the following system of equations

$$
\left[E\left(z_{i}\right)\left(y_{i}^{\prime}-\lambda y_{i}\right)=N\left(z_{i}\right)\right]_{i=1}^{n}(\text { system } 1)
$$

that has as unknowns the $2 w+k$ coefficients of the polynomials $E, N$. Observe that the above system (with $\lambda$ as a parameter) is not homogeneous (due to the fact that $E$ is monic). Recall that all steps up to this point follow exactly the Berlekamp-Welch algorithm (modulo the unknown $\lambda$ value).

Now consider the slightly extended system below:

$$
\left[E^{\prime}\left(z_{i}\right)\left(y_{i}^{\prime}-\lambda y_{i}\right)=N\left(z_{i}\right)\right]_{i=1}^{n} \quad(\text { system } 2)
$$

where $E^{\prime}(x)$ is a non-monic polynomial that has the same properties as $E$ (i.e. $E^{\prime}$ and $E$ have the same roots). It follows that system 2 defined above is homogeneous with $2 w+k+1$ unknowns. Let $A_{2}[\lambda]$ be the $n \times(2 w+k+1)$-matrix of system 2 .

Due to condition $i$ of definition 2 the number of equations $n$ satisfies

$$
n \geq 2 w+k+1
$$

and thus system 2 has at least as many equations as unknowns.

Case 1 of the Attack. $\operatorname{rank}\left(A_{2}[0]\right)=2 w+k+1$ (i.e., $A_{2}[0]$ is of full rank). It follows that there are $2 w+k+1$ linearly independent equations in system 2 for $\lambda=0$ (and their locations can be recovered e.g. by Gaussian elimination). Without loss of generality let us assume that these are the equations on locations $1, \ldots, 2 w+k+1$. We eliminate the remaining $n-(2 w+k+1)$ equations from system 2 , to make it a square homogeneous system, and we call the remaining equations system 3 .

It follows that if we substitute the value $\alpha$ for $\lambda$ in the matrix of the system 3 , the matrix is singular since it accepts a solution (the polynomials $E^{\prime}, N$ ) that is non-trivial. As a result the matrix of system 3 , denoted by $A_{3}[\lambda]$, has the following property:

$$
\exists \alpha \in \mathbb{F}: \operatorname{det}\left(A_{3}[\alpha]\right)=0
$$

Now observe that the determinant of system 3 is a polynomial $f(\lambda):=$ $\operatorname{det}\left(A_{3}[\lambda]\right)$ that is of degree at most $w+1$ (because $\lambda$ is only involved in the part of the matrix of system 3 that corresponds to the polynomial $\left.E^{\prime}\right)$.

Further observe that $f(0)=\operatorname{det}\left(A_{3}[0]\right) \neq 0$ because of our selection of $A_{3}[\lambda]$ to have the property that $A_{3}[0]$ is the full rank minor of the matrix $A_{2}[0]$. Thus, the value $\alpha$ is among the $w+1$ roots of $f$ and the output will be the list of roots of $f$. It follows that the above algorithm gives an efficient solution for the CAP problem. 
Case 2 of the Attack. $\operatorname{rank}\left(A_{2}[0]\right)<2 w+k+1$. In this case one can find a non-trivial solution of the system $A_{2}[0]$ which defines two non-zero polynomials $E^{\prime}, N$ such that

$$
\left[E^{\prime}\left(z_{i}\right) y_{i}^{\prime}=N\left(z_{i}\right)\right]_{i=1}^{n}
$$

Since $y_{i}^{\prime}=\alpha\left(p\left(z_{i}\right)+z_{i}^{k}+E_{i}\right)+p_{m s g}\left(z_{i}\right)+e_{i}$ it follows that

$$
\left[E^{\prime}\left(z_{i}\right)\left(\alpha\left(p\left(z_{i}\right)+z_{i}^{k}+E_{i}\right)+p_{m s g}\left(z_{i}\right)+e_{i}\right)=N\left(z_{i}\right)\right]_{i=1}^{n}
$$

Let $I$ be the subset of $\{1, \ldots, n\}$ for which it holds that $i \in I \Longleftrightarrow\left(e_{i}=\right.$ $0) \wedge\left(E_{i}=0\right)$. It follows that

$$
\left[E^{\prime}\left(z_{i}\right) p^{*}\left(z_{i}\right)=N\left(z_{i}\right)\right]_{i \in I}
$$

where $p^{*}(x)=\alpha\left(p(x)+x^{k}\right)+p_{m s g}(x)$. Recall that the degree of the polynomial $N$ is less than $k+w$ and $E^{\prime}$ is a polynomial of degree $w$; it follows that $E^{\prime}(x) p^{*}(x)$ is a polynomial of degree $w+k$.

Observe that $|I|$ is a random variable (denoted by $\eta$ ) ranging from $n-w-W$ to $n-\max \{w, W\}$. Next consider this relation:

$$
\eta>w+k \quad \text { (Sufficient Condition for Case 2) }
$$

Under the above relation, it follows that $|I| \geq w+k+1$ and as a result the polynomials $E^{\prime}(x) p^{*}(x)$ and $N(x)$ are equal. It follows immediately that $p^{*}=\frac{N}{E^{\prime}}$; naturally given $p^{*}$ we recover $\alpha$ immediately and non-ambiguously (in fact, in this case we will even be able to recover the value of the secret-key).

Performing a worst-case analysis of the above, we know that $\eta \geq n-w-W$ and as a result the attack would go through as long as $n-w-W>w+k \Longleftrightarrow n>$ $2 w+W+k$ something that matches condition \#1 of the AF03-cryptosystem (cf. section 2.1) and thus the case 2 of the attack can be carried for the parameters of the cryptosystem (without even taking into account that $\eta$ would be somewhat larger than its lower bound $n-w-W)$.

On the other hand, it would be of interest to us to find a necessary condition for case 2 of the attack (the reason for this will become clear in section 3). This can be found by setting $\eta$ to its highest possible value and requiring this to be greater than $w+k: \eta:=n-\max \{w, W\}>w+k$; this is equivalent to:

$$
n>w+\max \{w, W\}+k \text { (Necessary condition for Case 2) }
$$

\section{The Increased Error Case}

The cryptosystem of AF03 mandates that the number of errors introduced by the sender in the formation of the ciphertext is less or equal to $\frac{n-W-k-1}{2}$ (condition \#1 of section 2.1), to ensure unique decoding in the reduced PRinstance that is obtained after removing the $W$ locations that contain the errors of the Public-Key. 
We observe that the bound on $w$ is unreasonably low, for the following reason: many of the errors introduced by the sender will fall into the error-area of the public-key, and thus they will not affect the decryption operation (i.e., introducing a new error in an already erroneous location is a case where $1+1=1$ ).

To see this better, we can think of the sender in the cryptosystem to be playing the following game: he selects $w$ points out of $n$ and randomizes them. Since $W$ of these points will be discarded by the receiver it follows that the number of the good points (out of the total $n-W$ of good points) that will be randomized by the sender follow a hypergeometric distribution with mean value $\frac{n-W}{n}$. It follows that the expected number of good points that will be randomized by the sender are $w \frac{n-W}{n}$.

In order to ensure decoding for the decryption operation it suffices to force $\tilde{e} \leq \frac{n-W-k-1}{2}$ where $\tilde{e}$ is a random variable that follows the hypergeometric distribution with mean $\frac{n-W}{n}$. Let $w=\frac{1}{\frac{n-W}{n}+\epsilon} \frac{n-W-k-1}{2}$, for some $\epsilon>0$. Using the Chvátal bound for the hypergeometric distribution, Chv79, we have that

$$
\operatorname{Prob}\left[\tilde{e}>\left(\frac{n-W}{n}+\epsilon\right) w\right] \leq e^{-2 \epsilon^{2} w} \Longrightarrow \operatorname{Prob}\left[\tilde{e}>\frac{n-W-k-1}{2}\right] \leq e^{-2 \epsilon^{2} w}
$$

From the above, as long as $\epsilon<W / n$, if we set $w=\frac{1}{\frac{n-W}{n}+\epsilon} \frac{n-W-k-1}{2}$ it follows that the probability $\operatorname{Prob}\left[\tilde{e}>\frac{n-W-k-1}{2}\right] \leq e^{-2 \epsilon^{2} w}$, and thus condition \# 1 of section 2.1 will be satisfied in the probabilistic sense and decryption will succeed with probability $1-e^{-2 \epsilon^{2} w}$.

We will concentrate on parameters s.t. $W>w$ and $w$ is selected as above. Consider for example the assignment $n=2000, k=100, W \geq 1556$ (to avoid an attack with GS98 on the public-key), e.g. we set $W=1600$, and $\epsilon=1 / 6$; now observe that $W / n=0.8>1 / 6$. The equation for $w$ mentioned above yields $w=407$. It follows that the probability of correct decryption is $1-e^{-2 \frac{407}{36}}=$ $1-e^{-22} \approx 1-2^{-31}$. Observe now that case 2 of Coron's attack would be foiled since the necessary condition fails:

$$
n>w+\max \{w, W\}+k \Longleftrightarrow 2000>1600+407+100 \Longleftrightarrow \text { false }
$$

Thus, by merely increasing the number of errors that the sender of the cryptosystem introduces during encryption (relying on randomization to allow decryption with very high probability), we are capable of thwarting the analysis of Coron's attack (in particular the analysis of case 2 of the attack). Observe that this is possible without any other modification of the cryptosystem whatsoever.

Nevertheless, this is only a temporary comfort as we will prove in the next section.

\section{With High Probability Modified Coron's Attack Succeeds Against Increased Errors}

Next, we use another probabilistic analytical tool to show that, in fact, in spite of the increased errors, the attack actually works with high probability. 
First, observe the error-increase we introduced in section 3 does not apply to case 1 of Coron's attack. Indeed, one can show for any $\epsilon>0$ that

$$
w=\frac{1}{\frac{n-W}{n}+\epsilon} \frac{n-W-k-1}{2} \leq \frac{n-k-1}{2}
$$

and the condition $w \leq(n-k-1) / 2$ is sufficient for case 1 to go through (that is, if we can apply it). Recall that case 1 of the attack only applies to the case $\operatorname{det}\left(A_{3}[0]\right) \neq 0$.

We will show that this in fact happens most of the times (a fact observed in practice in [Cor03c] but not proved). This means that the attack works even in the increased error setting of the previous section. Let us recall the matrix of system 2 , as defined in section 2.3 .

$$
\begin{aligned}
& A_{2}[\lambda]=\left(B_{2} C_{2}[\lambda]\right)=
\end{aligned}
$$

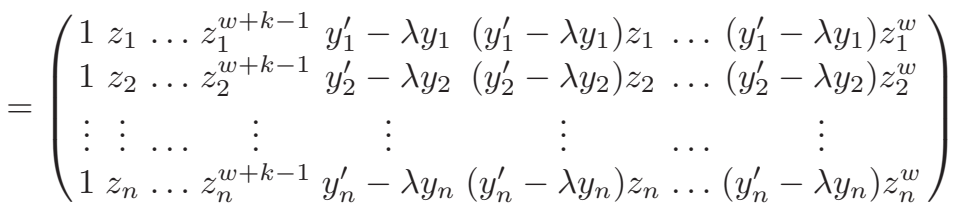

where $B_{2}$ is a Vandermonde matrix of dimension $w+k$ over the elements $z_{1}, \ldots, z_{n} ; B_{2}$ corresponds to the coefficients of $N(x) ; C_{2}[\lambda]$ is a Vandermonde matrix of dimension $w+1$ over the elements $z_{1}, \ldots, z_{n}$ where its $i$-th row is multiplied by $y_{i}^{\prime}-\lambda y_{i}$, for $i=1, \ldots, n ; C_{2}$ corresponds to the coefficients of $E^{\prime}(x)$. Recall that $A_{2}[\lambda]$ is a $n \times(2 w+k+1)$ matrix. We would like to prove that $\operatorname{rank}\left(A_{2}[0]\right)=2 w+k+1$ with overwhelming probability.

If $\operatorname{rank}\left(A_{2}[0]\right)<2 w+k+1$ then it follows that any $(2 w+k+1)$-minor of $A_{2}[0]$ is singular. Below we show that this event can only happen with very small probability (assuming that the underlying finite field $\mathbb{F}$ is large - something that is assumed in $\mathrm{AF} 03$ ) thus we deduce that the first case of the attack would work almost always.

Theorem 1. Let $\mathbf{P}=\operatorname{Prob}\left[\operatorname{rank}\left(A_{2}[0]\right)<2 w+k+1\right]$ be the probability that the rank of $A_{2}[0]$ is less than $2 w+k+1$ where the probability is taken over all possible choices for the given CAP instance out of which we construct $A_{2}[\lambda]$. It holds that $\mathbf{P} \leq 2 w /|\mathbb{F}|$ and the proof works even if the first inequality of condition $i$ of definition 2 is relaxed to only $w \leq(n-k-1) / 2$.

\section{The Most General AF System Avoids Coron's Attack}

\subsection{An "Optimal Variant" of the Cryptosystem}

In this section we show that the number of errors $w$ introduced by the sender can, in fact, be increased further beyond the improved bound that we describe in section 3 , by employing the proper decoding method for decryption (cf. figure 
11). In particular, we make the following crucial observation: AF03 requires that $w$ is below the error-correction bound of Reed-Solomon Codes, so that the decryption (decoding) is unique. Nevertheless the introduction of random errors in a large enough finite field (such fields are utilized in AF03]) suggests that uniqueness of decoding can be ensured far beyond the error-correction bound.

In the lemma below we show that randomly selected PR instances that can accept two different decodings are unlikely. This probabilistic analysis allow us to resort to modern list-decoding techniques in the sequel.

Lemma 1. Let $\left\{\left\langle z_{i}, y_{i}\right\rangle\right\}_{i=1}^{n}$ be a RS-Code codeword of a random message $p \in$ $\mathbb{F}[x]$ with degree $(p)<k$ that has e errors uniformly random distributed over $\mathbb{F}$, s.t. $e<n-k$. Then, the probability that it accepts another decoding $p^{\prime} \in \mathbb{F}[x]$ with $p \neq p^{\prime}$ is at most $\left(\begin{array}{c}n \\ t\end{array}\right)^{2} /\left(|\mathbb{F}|^{n-e-k}\right.$ ) (the probability is taken over all possible messages and noise corruptions).

Now observe that if the "message rate" is $\kappa:=k / n$ and the "error-rate" is $\epsilon:=e / n$, with $\kappa, \epsilon \in \mathbf{Q}^{+}$then it follows that the probability in lemma 1 is less than $\frac{4^{n}}{|\mathbb{F}|^{(1-\epsilon-\kappa) n}}$. As a result, provided that $\mathbb{F}$ satisfies $|\mathbb{F}|^{1-\epsilon-\kappa}>4$ it follows that the probability of proposition 1 is "negligible."

\section{Optimal Parameter Setting and Modifications for the Cryptosystem} of AF03. Taking advantage of the above Lemma in conjunction with the observation of section 3, we can increase the error-parameter $w$ further. We refer to our choice as optimal with respect to figure 1 under the basic assumption that the list-decoding algorithm of GS98 represents the state of the art in RSdecodability.

Below we assume that the sender employs the algorithm of GS98 for decryption. For this algorithm to work it should hold that $\tilde{e}<(n-W)-\sqrt{(n-W) k}$ where $\tilde{e}$ is the number of errors introduced in the area of good points of the public-key due to the encryption operation. As argued in section $3, \tilde{e}$ is a random variable following a hypergeometric distribution with mean $w \frac{n-W}{n}$. In our analysis below we will simply substitute $\tilde{e}$ for the expected number of errors. Note that this does not guarantee that the receiver will be capable of recovering the transmitted message "most of the times." To guarantee this we would have to show that the probability $\operatorname{Prob}[\tilde{e}<(n-W)-\sqrt{(n-W) k}]$ is overwhelming (as we did in section 3), something that cannot simply be inferred from the fact that the mean of $\tilde{e}$ is less than $(n-W)-\sqrt{(n-W) k}$; in order for the receiver to be able to decrypt most of the times we would instead require that the mean of $\tilde{e}$ is sufficiently lower than the bound $(n-W)-\sqrt{(n-W) k}$ and then employ the Chvátal bound on the tails of the hypergeometric distribution to bound the error probability by a negligible fraction, Chv79, (as in section 3).

Nevertheless, since we intend to cryptanalyze the resulting cryptosystem, we will opt for simply substituting $\tilde{e}$ for its mean, as this would only make our attack stronger. On the other hand observe that a public-key cryptosystem that works, say, half the times is still quite useful. Thus, substituting $\tilde{e}$ for $w \frac{n-W}{n}$ we obtain 


$$
w \frac{n-W}{n}<(n-W)-\sqrt{(n-W) k} \Longrightarrow w<n-n \sqrt{\frac{k}{n-W}}
$$

We conclude that the optimal selection would allow the parameter $w$ to be selected as high as:

$$
w<n\left(1-\sqrt{\frac{k}{n-W}}\right)
$$

The new bound above increases the number of errors that we can allow the sender to introduce, as long as $W$ is selected appropriately:

Proposition 2. There are choices for $W$ such that $W \geq n-\sqrt{n(k-1)}$ and $n\left(1-\sqrt{\frac{k}{n-W}}\right)>\frac{n-k-1}{2}$, as long as $k \geq 5$ and $k / n \leq 1 / 16$.

Now recall that the necessary condition for Coron's attack (both cases) is $w \leq \frac{n-k-1}{2}$. It follows from the proposition above that our analysis puts the parameter $w$ beyond the range of Coron's attack, provided that $W$ is properly selected. To illustrate this concretely, suppose that $n=2500$ and $k=101$. Then, $W$ should be selected in the range $[2000, \ldots, 2126]$; if we make the choice $W=2063$ then we can set $w$ to be as high as 1298, whereas Coron's attack would correct any value of $w$ only up to 1199 . Note that the gap of 99 elements between the bound of Coron's attack and the assignment $w=1298$ ensures that the application of the attack by removing 99 points at random would only succeed with probability less than $(0.52)^{99} \approx 2^{-96}$ (since the ratio of the senderintroduced error points is $\approx 0.52$ ).

Corollary 1. Coron's attack cannot be applied against the [AF03-cryptosystem in the optimal parameter setting.

To draw a parallel to our exposition in section 2.2, we introduce the problem $\mathrm{CAP}+$ to stand for the ciphertext-only attack problem of the optimal variant of Augot and Finiasz Cryptosystem, (the only difference from CAP being in the choice of $w)$ :

Definition 3 Ciphertext-Only Attack Problem in the Optimal Parameter Setting $(C A P+)$. Given two sequences of tuples $X_{1}:=\left\{\left\langle z_{i}, y_{i}\right\rangle\right\}_{i=1}^{n}$ and $X_{2}:=\left\{\left\langle z_{i}, y_{i}^{\prime}\right\rangle\right\}_{i=1}^{n}$ and parameters $k, w, W$ that satisfy the following conditions

i. $w<n\left(1-\sqrt{\frac{k}{n-W}}\right)$, and $W \geq n-\sqrt{n(k-1)}$.

ii. $\left\{\left\langle z_{i}, y_{i}-z_{i}^{k}\right\rangle\right\}_{i=1}^{n}$ is a random $P R$-instance with parameters $[n, k, W]$.

iii. $\exists \alpha \in \mathbb{F}$ such that $\left\{\left\langle z_{i}, y_{i}^{\prime}-\alpha y_{i}\right\rangle\right\}_{i=1}^{n}$ is a random PR-instance with parameters $[n, k, w]$.

Goal. Find a list of values of polynomial-length that contains the value $\alpha$. 
As before we show that any algorithm that solves $\mathrm{CAP}+$ can be used to mount a ciphertext-only attack on the cryptosystem of AF03 (but now in the optimal parameter setting):

Proposition 3. Let $\mathcal{A}$ be an algorithm that solves $C A P+$ in polynomial-time. Then any message encrypted in the cryptosystem of [AF03] in the optimal parameter setting can be decrypted without knowledge of the secret-key in polynomialtime in the security parameter.

In the Lemma below we give an upper bound on the value of $w$ (that is independent of $W$ ).

Lemma 2. For any $C A P+$ instance, it holds that $n-w>\sqrt[4]{n^{3}(k-1)}$.

\section{The Attack Against the General System Employing List-Decoding}

The results we present in this section (essentially an algorithm for solving $\mathrm{CAP}+$ ) is based on Sudan's list-decoding algorithm, Sud97 and Guruswami Sudan GS98 algorithms (for both there are efficient polynomial-time algorithms, see [McE03]).

\subsection{The Attack}

Let $n, k, w, W \in \mathbf{Z}$ and $X_{1}=\left\{\left\langle z_{i}, y_{i}\right\rangle\right\}_{i=1}^{n}, X_{2}=\left\{\left\langle z_{i}, y_{i}^{\prime}\right\rangle\right\}_{i=1}^{n}$ be an instance of $\mathrm{CAP}+$. We denote $\hat{y}_{i}:=y_{i}^{\prime}-\lambda y_{i}$ for $i=1, \ldots, n$, where $\lambda$ is an unspecified parameter (free variable); to set the parameter $\lambda$ to a specific value $\alpha$ we will write $\hat{y}_{i}[\alpha]$.

According to the definition of a CAP + instance we know that there exists a value $\alpha \in \mathbb{F}$ (the "encryption coefficient") and a polynomial $p \in \mathbb{F}[x]$ of degree less than $k$ (the "message polynomial") that agrees with $n-w$ of the points $\left\langle z_{i}, \hat{y}_{i}[\alpha]\right\rangle$. Define $l:=n-w-1$. Next we consider the following system of equations on a set of unknowns $\left\{q_{j_{1}, j_{2}}\right\}_{j_{1} \geq 0, j_{2} \geq 0, j_{1}+(k-1) j_{2}<l}$ (called system 4 ):

$$
\forall i \in\{1, \ldots, n\} \quad \sum_{j_{1} \geq 0, j_{2} \geq 0, j_{1}+(k-1) j_{2}<l} q_{j_{1}, j_{2}} z_{i}^{j_{1}} \hat{y}_{i}^{j_{2}}=0 \text { (system 4) }
$$

Observe that any solution to system 4 above defines a bivariate polynomial $Q(x, y)$ that satisfies the property $\operatorname{degree}_{Q, x}+(k-1) \operatorname{degree}_{Q, y}<l$.

Lemma 3. The number of unknowns of system 4, is at least $\frac{l(l-1)}{2(k-1)}$.

Recall that from proposition 2 we know that we only consider parameter choices that satisfy $k / n \leq 1 / 16$. For such range of parameters (and sufficiently large $n$ ) we, in fact, show:

Lemma 4. System 4 is not overdefined provided that $n \geq 19$ and $k / n \leq 1 / 9$. 
Subsequently we omit the appropriate number of unknowns from system 4, to equalize the number of unknowns and equations. This results in a square homogeneous system of $n$ equations and unknowns that we call system 5 . We denote the matrix of system 5 by $A[\lambda]$.

Theorem 2. Let $\alpha \in \mathbb{F}$ be the "encryption coefficient" for a CAP+ instance as defined in item iii of definition [3. The matrix $A[\alpha]$ as constructed above is singular.

Since $A[\alpha]$ is singular, it follows that if we define the polynomial $f(\lambda):=$ $\operatorname{Det}(A[\lambda]), \alpha$ will be among the solutions of $f(\lambda)$. Thus we can solve CAP + by computing all the (polynomialy many, by degree constraint) roots of $f(\lambda)$.

Theorem 3. The probability $\mathbf{P}$ that the polynomial $f(\lambda)=\operatorname{Det}(A[\lambda])$ is the zero-polynomial satisfies $\mathbf{P} \leq 2 s(n-l) /|\mathbb{F}|$, where $s=\left\lfloor\frac{l-1}{k-1}\right\rfloor$ (the maximum degree of the $y$-variable in any of the columns of $A[\lambda])$.

\section{$7 \quad$ Summary}

In this section, we summarize our cryptanalytic results.

Given an instance of $\mathrm{CAP}+\left\{\left\langle z_{i}, y_{i}\right\rangle\right\}_{i=1}^{n},\left\{\left\langle z_{i}, y_{i}^{\prime}\right\rangle\right\}_{i=1}^{n}$ with parameters $n, k, w, W$. 0 . Set $l:=n-w-1$.

1. Select $D \subseteq \mathbb{N} \times \mathbb{N}$ so that $|D|=n$ and for all $\left\langle j_{1}, j_{2}\right\rangle \in D, j_{1}+(k-1) j_{2}<l$

2. Let $\boldsymbol{D}=\left\langle\left\langle j_{1}[1], j_{2}[1]\right\rangle, \ldots,\left\langle j_{1}[n], j_{2}[n]\right\rangle\right\rangle$, a lexicographic ordering of $D$.

3. Construct a $(n \times n)$-matrix $A$ so that its $\left(i, i^{\prime}\right)$-entry equals $z_{i}^{j_{1}\left[i^{\prime}\right]}\left(y_{i}^{\prime}-\lambda y_{i}\right)^{j_{2}\left[i^{\prime}\right]}$

4. Compute $f(\lambda):=\operatorname{det}(A[\lambda])$ symbolically to obtain the polynomial $f$ on $\lambda$.

5. Output all roots of $f$.

Fig. 2. The algorithm that solves CAP+

First in figure 2 we overview the $\mathrm{CAP}+$ algorithm that was presented in the previous section. Using this, the general cryptosystem based on Augot and Finiasz AF03, even under the optimal choice of parameters is broken under ciphertext-only attacks. The breaking algorithm is summarized in figure 3

Given the public-key and a ciphertext of the AF03-cryptosystem with parameters $n, k, w, W$.

1. if $w \leq \frac{n-k-1}{2}$ invoke case 1 of Coron's attack.

2. else invoke the CAP + algorithm of figure 1, and recover the plaintext using Guruswami-Sudan algorithm (as described in proposition 33).

Fig. 3. The attack against the Generalized Version of AF03-Cryptosystem 
Note that the attack outlined above is probabilistic and is guaranteed to work with very high probability as we have shown in theorem 1 (for case 1 of Coron's attack), and theorem 3 (for CAP + algorithm).

\section{References}

[AF03] Daniel Augot and Matthieu Finiasz, A Public Key Encryption Scheme Based on the Polynomial Reconstruction Problem, Eli Biham (Ed.): Advances in Cryptology - EUROCRYPT 2003, International Conference on the Theory and Applications of Cryptographic Techniques, Warsaw, Poland, May 4-8, 2003, Proceedings. Lecture Notes in Computer Science 2656 Springer 2003, pp. 229-240.

[AFL03] Daniel Augot, Matthieu Finiasz and Pierre Loidreau, Using the Trace Operator to repair the Polynomial Reconstruction based Cryptosystem presented at Eurocrypt 2003, Cryptology ePrint Archive, Report 2003/209, 2003, http://eprint.iacr.org/

[BW86] Elwyn R. Berlekamp and L. Welch, Error Correction of Algebraic Block Codes. U.S. Patent, Number 4,633,470, 1986.

[Chv79] Vasek Chvátal, The tail of the hypergeometric distribution, Discrete Math, Vol. 25, pp. 285-287, 1979.

[Cor03a] Jean-Sebastien Coron, Cryptanalysis of a public-key encryption scheme based on the polynomial reconstruction problem, Cryptology ePrint Archive, Report 2003/036. http://eprint.iacr.org/

[Cor03b] Jean-Sebastien Coron, Cryptanalysis of the Repaired Public-key Encryption Scheme Based on the Polynomial Reconstruction Problem, Cryptology ePrint Archive, Report 2003/219. http://eprint.iacr.org/

[Cor03c] Jean-Sebastien Coron, Cryptanalysis of a public-key encryption scheme based on the polynomial reconstruction problem, in Feng Bao, Robert H. Deng, Jianying Zhou (Eds.): Public Key Cryptography - PKC 2004, 7th International Workshop on Theory and Practice in Public Key Cryptography, Singapore, March 1-4, 2004. Lecture Notes in Computer Science 2947 Springer 2004, pp. 14-27.

[GS98] Venkatesan Guruswami and Madhu Sudan, Improved Decoding of ReedSolomon and Algebraic-Geometric Codes. In the Proceedings of the 39th Annual Symposium on Foundations of Computer Science, Palo Alto, California, November 8-11, IEEE Computer Society, pp. 28-39, 1998.

[KY02] Aggelos Kiayias and Moti Yung, Cryptographic Hardness based on the Decoding of Reed-Solomon Codes, in the Proceedings of ICALP 2002, Lecture Notes in Computer Science, vol. 2380, Malaga, Spain, July 8-13, pp. 232-243.

[KY04] Aggelos Kiayias and Moti Yung, Cryptanalyzing the PolynomialReconstruction based Public-Key System Under Optimal Parameter Choice, Cryptology ePrint Archive, Report 2004/217. http://eprint.iacr.org/

[McE78] Robert J. McEliece. A public key cryptosystem based on algebraic coding theory. Jet Propulsion Lab, DSN Progress Report, 42(44), pp. 114-116, Jan-Feb 1978. 
[McE03] Robert J. McEliece, The Guruswami-Sudan Decoding Algorithm for Reed-Solomon Codes, IPN Progress Report 42-153, May 15, 2003. http : //ipnpr.jpl.nasa.gov/tmo/progress_report/42 - 153/153F.pdf.

[Sch80] Jacob T. Schwartz, Fast Probabilistic Algorithms for Verifications of Polynomial Identities, Journal of the ACM, Vol. 27(4), pp. 701-717, 1980.

[Sud97] Madhu Sudan, Decoding of Reed Solomon Codes beyond the Error-Correction Bound. Journal of Complexity 13(1), pp. 180-193, 1997. 